\title{
Boiler-Turbine Control System Design Using a Genetic Algorithm
}

\author{
Robert Dimeo, Student Member, IEEE and Kwang Y. Lee, Senior Member, IEEE \\ Department of Electrical Engineering \\ The Pennsylvania State University \\ University Park, PA 16802
}

\begin{abstract}
This paper discusses the application of a genetic algorithm to control system design for a boiler-turbine plant. In particular we study the ability of the genetic algorithm to develop a proportional-integral (PI) controller and a state feedback controller for a. non-linear multiinput/multi-output (MIMO) plant model. The plant model is presented along with a discussion of the inherent difficulties in such controller development. A sketch of the genetic algorithm (GA) is presented and its strategy as a method of control system design is discussed. Results are presented for two different control systems that have been designed with the genetic algorithm.
\end{abstract}

Keywords - Genetic Algorithm, Boiler-Turbine Control, Optimal Control, PI Control

\section{INTRODUCTION}

A practical control problem that has received a great deal of attention lately, is the robust control of power plants [1]. It is well known that automatic control is deemed a necessary condition for safe operation which minimizes material fatique, the number of staff, and enables efficient plant management [2]. However, the design of controllers for power plants is not a trivial task. The challenge in controller design for these plants exists because they are typically non-linear and multivariable with multiple control objectives. While conventional controls such as PID compensators yield an acceptable response, they do not have the flexibility necessary to provide a good performance over a wide region of operation. Application of modern optimal control techniques yields system performances that are optimal at only one operating point.

\footnotetext{
95 SM 450-7 EC A paper recommended and approved by the IEEE Energy Development and Power Generation Committee of the IEEE Power Engineering society for presentation at the 1995 IEEE/PES Summer Meeting. July 23-27, 1995, Portland, OR. Manuscript submitted July 27, 1994; made available for printing April 27,1995.
}

Recent applications of robust control to power plant models have yielded quite favorable results [1], [3]. These robust controllers perform very well over a wide range of operation. However, these robust control design methodologies require frequency response information in addition to a linearized model of the plant. A goal of modern intelligent control design is to obtain a controller based on input/output information only. The genetic algorithm (GA) is a method that holds promise for such a control system design.

The GA is a search technique based on the mechanics of natural genetics and survival-of-the-fittest. Touted as an efficient and general method of searching a complex space [4], the GA has had success in many areas. The GA has proven successful in obtaining a solution to the traveling salesman problem [5], optimal control of an aircraft autopilot system [6], multivariate curve-fitting, and game-playing [5]. A number of these achievements suggest the potential utility of the GA as a method for control system design. In this paper we explore this potential.

This paper is divided into 5 sections. In section II the non-linear, multi-input/multi-output (MIMO) boiler-turbine model is presented. Section III presents the principal ideas underlying the use of the GA as a method for control system design. Section IV presents the results of two control structures that are designed using the GA. Finally, section V contains the main conclusions of this paper.

\section{THE BOILER-TURBINE MODEL}

The boiler-turbine model used in this paper was first developed by Bell and Åström [7]. The model is a $3^{\text {rd }}$ order, non-linear MIMO system with hard constraints and rate limits imposed on the actuators.

\section{A. The Non-Linear Model}

The model is based on the boiler-turbine plant P16/G16 at the Sydvenska Kraft AB Plant in Malmö, Sweden. The boiler is oil-fired and the rated power is $160 \mathrm{MW}$. Data acquired during a series of experiments in 1969 form the basis for the system identification. Both physics and empirical methods were used to produce this boiler-turbine dynamic model.

Since 1969, the model has undergone a number of alterations. Subsequent improvements in the plant model have resulted in better models that yield improved predictive 
abilities for the plant. Aström and Eklund originally used test data from the 1969 data logs to perform crude parameter estimation used in their model [8]. This resulted in a $2^{\text {nd }}$ order non-linear system of differential equations with fuel flow and control valve setting as the control variables and drum pressure and power output as the output variables. In 1977, Morton and Price updated the boiler model in an attempt to include drum water level deviation by introducing evaporation rate dynamics [9]. Their model did not include power output, though, and the water level prediction was poor for certain inputs. Their model was extended to include the power, and Bell and Åström combined it with their work to produce a $3^{\text {rd }}$ order non-linear MIMO model with fuel flow, control valve position, and feedwater flow as control inputs, and drum pressure, power output, and drum water level deviation as outputs. It was found that the inclusion of the evaporation equation and fluid density dynamics in Bell and Åström's model yielded a reasonable depiction of the drum water level dynamics. Although the model is of low order, it is capable of illustrating some of the complex dynamics associated with the real plant [7]. The dynamics for the system are [8]

$$
\begin{aligned}
& \frac{d p}{d t}=-0.0018 u_{2} p^{\frac{9}{8}}+0.9 u_{1}-0.15 u_{3} \\
& \frac{d P_{o}}{d t}=\left(0.073 u_{2}-0.016\right) p^{\frac{9}{8}}-0.1 P_{o} \\
& \frac{d \rho_{f}}{d t}=\frac{\left(141 u_{3}-\left(1.1 u_{2}-0.19\right) p\right)}{85}
\end{aligned}
$$

where $p=$ drum pressure $\left(\mathrm{kg} / \mathrm{cm}^{2}\right), P_{o}=$ power output $(\mathrm{MW})$, and $\rho_{f}=$ fluid density $\left(\mathrm{kg} / \mathrm{m}^{3}\right)$. The normalized inputs to the system are $u_{1}=$ fuel flow valve position, $u_{2}=$ steam control valve position, and $u_{3}=$ feedwater flow valve position. The following limitations are imposed on the valves:

$$
\begin{gathered}
\left|\frac{d u_{1}}{d t}\right| \leq 0.007 / \mathrm{sec} \\
-2 / \mathrm{sec} \leq \frac{d u_{2}}{d t} \leq 0.02 / \mathrm{sec} \\
\left|\frac{d u_{3}}{d t}\right| \leq 0.05 / \mathrm{sec},
\end{gathered}
$$

and all valve position variables are constrained to lie in the interval $[0,1]$. The outputs to the system are $p$ (drum pressure), $P_{o}$ (output power), and $X_{w}$ (drum water level in meters). Pressure and power output are just the first two state variables, whereas water level is found through the auxiliary relationships

$$
X_{w}=0.05\left(0.13073 \rho_{f}+100 \alpha_{c s}+\frac{q_{e}}{9}-67.975\right)
$$

$$
\begin{gathered}
\alpha_{c s}=\frac{\left(1-0.001538 \rho_{f}\right)(0.8 p-25.6)}{\rho_{f}(1.0394-0.0012304 p)} \\
q_{e}=\left(0.854 u_{2}-0.147\right) p+45.59 u_{1}-2.514 u_{3}-2.096
\end{gathered}
$$

where $q_{e}$ is the evaporation rate $(\mathrm{kg} / \mathrm{s})$ and $\alpha_{c s}$ is the steam quality.

\section{B. The Linear Model}

The performance of the control systems designed with the GA will be compared to the linear quadratic regulator designed from the linearized model. At a load level of $66.65 \mathrm{MW}$, pressure of $108 \mathrm{~kg} / \mathrm{cm}^{2}$, and fluid density of $428 \mathrm{~kg} / \mathrm{m}^{3}$, the nominal inputs are found to be $u^{\circ}=\left[\begin{array}{lll}0.34 & 0.69 & 0.436\end{array}\right]^{T}$. From these nominal values, a linearized model is obtained from a truncated Taylor series expansion of the non-linear equations. The non-linear dynamics are of the form

$$
\begin{aligned}
& \frac{d x}{d t}=f(x, u) \\
& y=g(x, u),
\end{aligned}
$$

and linearization of the system about the nominal operating point, $\left(x^{o}, u^{o}\right)$, requires calculating the linear system matrices $\quad A=\left[\frac{\partial f}{\partial x}\right]_{\left(x^{o}, u^{o}\right)}, \quad B=\left[\frac{\partial f}{\partial u}\right]_{\left(x^{o}, u^{o}\right)}$, $C=\left[\frac{\partial g}{\partial x}\right]_{\left(x^{\circ}, u^{\circ}\right)}, \quad$ and $\quad D=\left[\frac{\partial g}{\partial u}\right]_{\left(x^{\circ}, u^{\circ}\right)}$ for $x^{o}=\left[\begin{array}{lll}108 & 66.65 & 428\end{array}\right]^{T}$ and $u^{o}=\left[\begin{array}{lll}0.34 & 0.69 & 0.436\end{array}\right]^{T}$. The linear approximation to the system is

$$
\begin{aligned}
& \frac{d \bar{x}}{d t}=A \bar{x}+B \bar{u} \\
& \bar{y}=C \bar{x}+D \bar{u},
\end{aligned}
$$

where $\bar{x}=x-x^{o}, \bar{y}=y-y^{o}$, and $\bar{u}=u-u^{o}$. The linear system matrices are found to be

$$
A=\left(\begin{array}{ccc}
-2.509 \times 10^{-3} & 0 & 0 \\
6.94 \times 10^{-2} & -0.1 & 0 \\
-6.69 \times 10^{-3} & 0 & 0
\end{array}\right),
$$

$$
B=\left(\begin{array}{ccc}
0.9 & -0.349 & -0.15 \\
0 & 14.155 & 0 \\
0 & -1.389 & 1.659
\end{array}\right)
$$




$$
\begin{aligned}
C & =\left(\begin{array}{ccc}
1 & 0 & 0 \\
0 & 1 & 0 \\
6.34 \times 10^{-3} & 0 & 4.71 \times 10^{-3}
\end{array}\right), \text { and } \\
D & =\left(\begin{array}{ccc}
0 & 0 & 0 \\
0 & 0 & 0 \\
0.253 & 0.512 & -0.014
\end{array}\right) .
\end{aligned}
$$

Table I shows the various operating points of the plant.

\section{THE GA CONTROL SYSTEM DESIGN}

In this section the GA is discussed and its application to control system design is presented.

\section{A. The Genetic Algorithm}

The GA used in this paper is very similar to the algorithm that can be found in the standard literature on the topic [10], [4], [11], [12], also known as the simple genetic algorithm. We used the three-operator GA with only minor deviations from the original.

In this scheme, an initial population of binary strings is created randomly. Each of these strings represents one possible solution to the search problem. This binary representation is certainly not unique but it is simple to implement. Next the solution strings are converted into their decimal equivalents and each candidate solution is tested in its environment. The fitness of each candidate is evaluated through some appropriate measure. The algorithm is driven towards maximizing this fitness measure. For example, in a function maximization problem the fitness measure might be the function evaluation itself. Application of the GA to an optimal control problem entails minimizing the integralsquared error (ISE) of the input and states. After the fitness of the entire population has been determined, it must be determined whether or not the termination criterion has been satisfied. This criterion can be any number of things. One possibility is to stop the algorithm at some finite number of generations and designate the result as the best fit from the population. Another possibility is to test if the average fitness of the population exceeds some fraction of the best fit in the population. If the criterion is not satisfied then we continue with the three genetic operators. Next, the three genetic operations of reproduction, crossover, and mutation are invoked. Fitness-proportionate reproduction is effected throught the simulated spin of a weighted roulette wheel.

The roulette wheel is biased with the fitnesses of each of the solution candidates. The wheel is spun $\mathbf{N}$ times where $\mathbf{N}$ is the number of strings in the population. This operation yields a new population of strings that reflect the fitnesses of the previous generation's fit candidates. The next operation, crossover, is performed on two strings at a time that are selected from the population at random. Crossover involves choosing a random position in the two strings and swapping the bits that occur after this position. In one generation the crossover operation is performed on a specified percentage of
TABLEI

Operating points for plant.

\begin{tabular}{|c|c|c|c|c|c|c|c|}
\cline { 2 - 8 } \multicolumn{1}{c|}{} & $\begin{array}{c}1 \\
70 \%\end{array}$ & $80 \%$ & $90 \%$ & $\begin{array}{c}\text { NOM } \\
100 \%\end{array}$ & $\begin{array}{c}4 \\
110 \%\end{array}$ & $\begin{array}{c}5 \\
120 \%\end{array}$ & $\begin{array}{c}6 \\
130 \%\end{array}$ \\
\hline$x_{1}^{o}$ & 75.60 & 86.40 & 97.20 & 108 & 118.8 & 129.6 & 140.4 \\
\hline$x_{2}^{o}$ & 15.27 & 36.65 & 50.52 & 66.65 & 85.06 & 105.8 & 128.9 \\
\hline$x_{3}^{o}$ & 299.6 & 342.4 & 385.2 & 428 & 470.8 & 513.6 & 556.4 \\
\hline$u_{1}^{o}$ & 0.156 & 0.209 & 0.271 & 0.34 & 0.418 & 0.505 & 0.6 \\
\hline$u_{2}^{o}$ & 0.483 & 0.552 & 0.621 & 0.69 & 0.759 & 0.828 & 0.897 \\
\hline$u_{3}^{o}$ & 0.183 & 0.256 & 0.340 & 0.435 & 0.543 & 0.663 & 0.793 \\
\hline
\end{tabular}

the population. This proportion of the population is specified at the initialization stage of the algorithm. The final genetic operator in the algorithm is mutation. Mutation is performed sparingly, typically every 100-1000 bit transfers from crossover, and it involves selecting a string at random as well as a bit position at random and changing it from a 1 to $a 0$ or vice-versa. After mutation, the new generation is complete and the procedure begins again with fitness evaluation of the population.

In a control system design using the $\mathrm{GA}$ the parameters that are represented as binary strings are the relevant control parameters. In the design of the proportional-integral (PI) control system, the parameters are the 12 proportional, integral, and cross-coupling gains in the coupled controller illustrated in Fig. 1. In the linear quadratic regulator (LQR) controller the parameters are the 9 state feedback gains in the standard $L Q R$ configuration. In each of the control designs the quadratic performance index is selected

$$
J=\int_{t_{o}}^{t_{f}}\left(\left(y-y^{\text {ref }}\right)^{T} Q\left(y-y^{\text {ref }}\right)+u^{T} R u\right) d t,
$$

and a fitness measure is designated as $f=1 /(1+J)$.

\section{B. PI Controller Design}

In the coupled PI controller design the gains are trained in 5 stages. The first stage consists of training the proportional gains in the PI controller only and leaving the others fixed at zero. The initial gains are selected at random between some coarse upper and lower bounds and tuned through each genetic iteration. Once some pre-specified convergence criterion has been achieved, the best fit triplet of proportional gains is designated as the result for the stage. Stage 2 extends the strings in the population to include the next three integral gains. The bounds on the first three values in the string (the proportional gains) are between $\pm 25 \%$ of the result from the previous stage. This constrains the first three gains to be around their previous best value, and yet allows fine-tuning with the introduction of the new integral gains. The integral 
gains are set initially to random values between some coarse upper and lower bounds and tuned through each genetic iteration. Again, this is executed until some convergence criterion has been attained. This procedure is repeated in the same manner with the string lengthened by two more bits to represent the coupling of the first input error to the other two. This is done two more times to train the final four coupling gains. The training cycle is tabulated in table II.

Each stage of training entails an alternating reference demand change in pressure and power. After the system has been trained to perform well with one reference change, it is then trained to perform well with a different reference demand change. This is done until some convergence criterion has been attained. By training the controller gains in this manner the gains are tuned to some average performance between two high-performance results. The goal of this method is a controller that performs well at different operating points.

A pressure demand change between $100 \%$ and $120 \%$ of the nominal operating point is used in the first training cycle while the other inputs are held fixed at their nominal operating points. This sequence is illustrated below.

$$
\begin{aligned}
y_{1}^{r e f}= & 108 \\
& +(118.8-108) 1^{+}(t-200)+(129.6-118.8) 1^{+}(t-600) \\
& +(118.8-129.6) 1^{+}(t-1000)+(108-118.8) 1^{+}(t-1400) \\
& y_{2}^{r e f}=66.65, y_{3}^{\text {ref }}=0
\end{aligned}
$$

TABLE II

Training schedule for coupled PI gains.

\begin{tabular}{|c|c|}
\hline Stage & Gains to Train \\
\hline I & $\mathrm{Kp} 1, \mathrm{Kp} 2, \mathrm{Kp} 3$ \\
\hline II & $\mathrm{Ki} 1, \mathrm{Ki} 2, \mathrm{Ki} 3$ \\
\hline III & $\mathrm{K} 12, \mathrm{~K} 13$ \\
\hline IV & $\mathrm{K} 21, \mathrm{~K} 23$ \\
\hline V & $\mathrm{K} 31, \mathrm{~K} 32$ \\
\hline
\end{tabular}

where $1^{+}(t)$ is a step function starting at $t=0$. The second demand change in the cycle is the power demand change. As in the pressure change, the power demand is changed between $100 \%$ and $120 \%$ of the nominal operating point while holding pressure and level fixed.

This demand is given below.

$$
\begin{gathered}
y_{2}^{\text {ref }}=66.65+(85.063-66.65) 1^{+}(t-200) \\
+(105.8-85.063) 1^{+}(t-600) \\
+(85.063-105.8) 1^{+}(t-1000) \\
+(66.65-85.063) 1^{+}(t-1400) \\
y_{1}^{r e f}=108, y_{3}^{r e f}=0 .
\end{gathered}
$$

The goal of the controller is to track step demands in power and pressure. To achieve this the following performance index is selected to be minimized:

$$
\mathrm{J}=\mathrm{J}_{\mathrm{o}}+\mathrm{J}_{\mathrm{ss}} \text {, }
$$

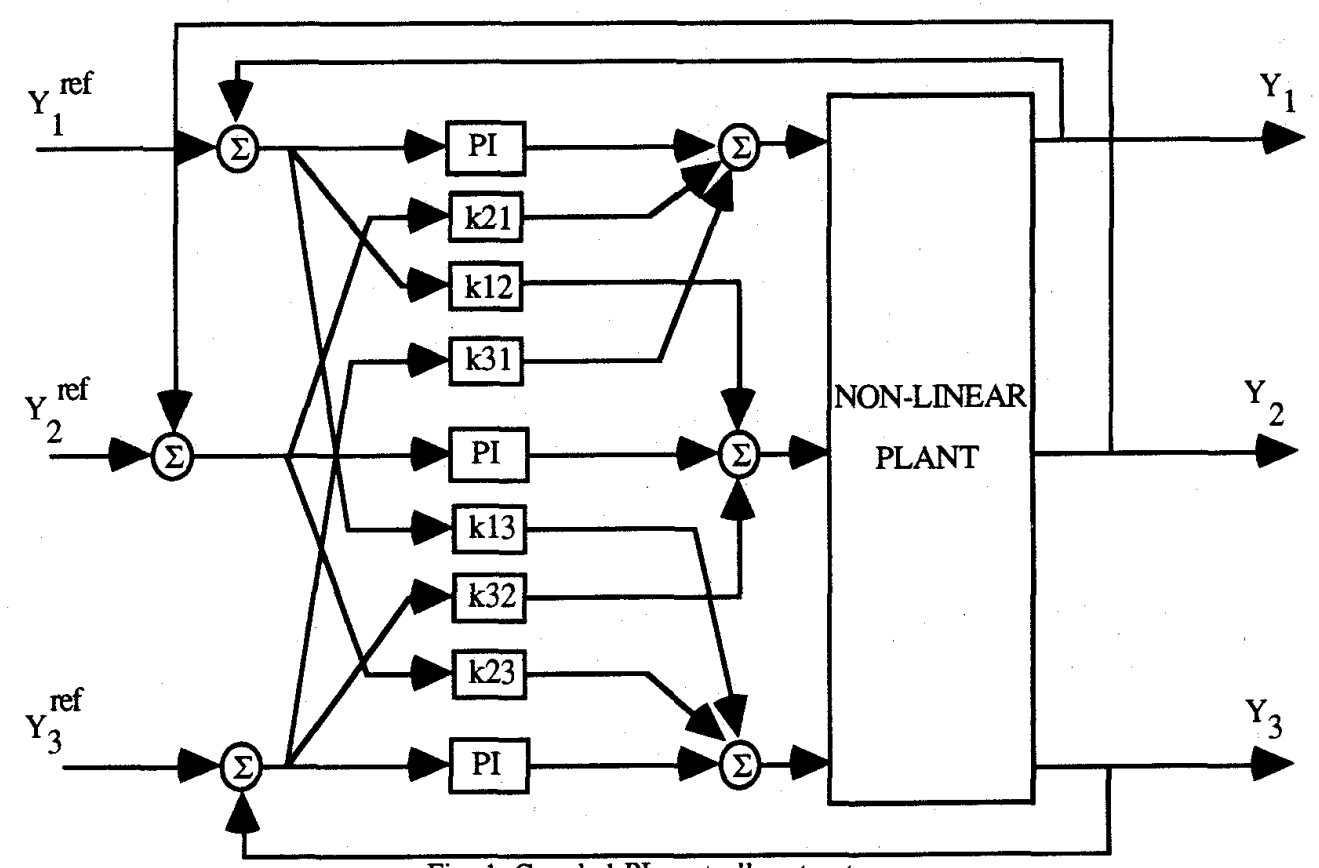

Fig. 1. Coupled PI controller structure. 
where $J_{o}$ is defined by (6). In addition, we penalize the steady-state error through computation of

$$
\mathrm{J}_{\mathrm{ss}}=\sum_{\mathrm{i}=1}^{3} \mathrm{e}_{\mathrm{i}}\left(\mathrm{t}_{\mathrm{f}}\right)
$$

where

$$
e_{i}(t)=y_{i}(t)-y_{i}^{\text {ref }}(t)
$$

The weighting matrices are selected as

$$
\mathrm{Q}=\mathrm{I}_{3 \times 3}, \mathrm{R}=\left(\begin{array}{ccc}
724 & 0 & 0 \\
0 & 141 & 0 \\
0 & 0 & 5
\end{array}\right)
$$

The values of these matrices can be determined from the relative importance of the variables and from the relationships of the outputs to the inputs in the steady-state. Since the order of the system has been increased by including the integral action in the controller, we weigh the steady-state error heavily in the hopes of achieving perfect steady-state tracking. cycle:

The following GA parameters are selected for the training

$$
\begin{array}{ll}
\text { Population size } & 64 \\
\text { Crossover rate } & 0.9 \\
\text { Mutation rate } & 0.001 \\
\text { Parameter resolution } & 9 \text { bits per substring }
\end{array}
$$

Each training cycle is executed until either 30 generations have elapsed or $95 \%$ convergence has been achieved. In this paper $95 \%$ convergence was the condition that was met first. Finally, the fitness function used in this training cycle is $10^{7} / \mathrm{J}$.

\section{LQR Controller Design}

The goal of the genetic algorithm is to determine the matrix gains in the feedback path to ensure tracking of the reference signal over a wide operating range. The controller structure is simply a state feedback matrix in the feedback path and a feedforward gain matrix to ensure tracking. This feedforward matrix is found using the transfer function from the input to the output [13]. To obtain reasonable tracking over a wide operating range, the GA trains the gains of the feedback matrix with a demand that covers the nominal load level as well as a large load change. We select a demand change in power while attempting to maintain drum pressure and drum water level. These demands are:

$$
\begin{gathered}
y_{1 \text { ref }}=108, \quad y_{3 \mathrm{ref}}=0, \\
y_{2 \text { ref }}=66.65+(105.8-66.65) \times 1^{+}(t-100) \\
+(129.89-105.8) \times 1^{+}(t-300) .
\end{gathered}
$$

The genetic algorithm is implemented with a number of different genetic parameters (i.e., crossover rate, mutation rate, population number, etc.). In an effort to improve convergence, the individual matrix elements' resolutions are all increased at a specified generation of the run. At this specified time, all of the substrings are lengthened by two bits. The old matrix element values are retained but at a greater precision. We select this lengthening of the bits to occur one-third of the way through the optimization run. We choose the following parameters:

$\begin{array}{ll}\text { Population size } & 100 \\ \text { Crossover rate } & 0.8 \\ \text { Mutation rate } & 0.2 \\ \text { Terminating generation } & 30 \\ \text { Matrix element interval } & {[-1,1]} \\ \text { Initial resolution } & 15 \text { bits per substring } \\ \text { Resolution after } 10 \text { gen. } & 17 \text { bits per substring }\end{array}$

In addition to these parameters, the initial population is seeded with 3 members from a previous run's "best fit" population with the remainder of the population constructed at random. This is another attempt to obtain quicker convergence.

In the GA optimization runs, the fitness function is selected as $100 /(1+J)$ where $J$ is identical to (6). In addition, the weighting matrices are identical to those in the PI controller design.

\section{GA DESIGN RESULTS}

The performance of each of the control systems is tested with the following inputs to the system,

$$
\begin{gathered}
y_{I_{\text {ref }}}(t)=108+(120-108) \times 1^{+}(t-200) \\
y_{2 \text { ref }}(t)=66.65+(120-66.65) \times 1^{+}(t-600) \\
y_{3 \text { ref }}(t)=0
\end{gathered}
$$

This reference change in pressure and power represents a relatively large demand change in both variables and illustrates the merits and weaknesses of each design.

\section{A. GA/PI Controller Results}

The performance of the system with the GA-designed coupled PI controller is illustrated in Figs. 2-4. Table III contains the controller gains at each stage of training. The drum pressure is very oscillatory with a large overshoot. This response settles out with a steady-state error of 0.175 . The power response is good during the first change in pressure at 200 seconds. As can be seen, the change has little influence on power. When the demand in power steps up to $120 \mathrm{MW}$ the power output overshoots by about $5 \mathrm{MW}$ for a duration of less than 200 seconds. This overshoot can be attributed to the saturation of the control valve for that period of time (not shown). Again, the change in power has little effect on pressure at 600 seconds. The steady-state error of 
the power is $0.0004 \mathrm{MW}$. The drum water level deviation is acceptable during both transitions in pressure and power. The largest deviation of the level occurs during the power demand

Table III

Gains at each training stage.

\begin{tabular}{|c|c|c|c|c|c|}
\hline & I & II & III & IV & V \\
\hline Kp1 & 11.3883 & 8.9675 & 10.2678 & 11.7566 & 11.1185 \\
\hline Kp2 & 0.0793 & 0.0009 & 0.0007 & 0.0005 & 0.004 \\
\hline Kp3 & 1.2051 & 0.9579 & 1.0423 & 1.1341 & 1.1631 \\
\hline Ki1 & - & 0.0041 & 0.0038 & 0.0036 & 0.0033 \\
\hline Ki2 & - & 0.0191 & 0.0149 & 0.0117 & 0.0093 \\
\hline Ki3 & - & 0.0269 & 0.0238 & 0.0211 & 0.0186 \\
\hline K12 & - & - & 0.0405 & 0.0386 & 0.0292 \\
\hline K13 & - & - & 0.0979 & 0.1214 & 0.1344 \\
\hline K21 & - & - & - & 0.0381 & 0.0468 \\
\hline K23 & - & - & - & 0.0950 & 0.0875 \\
\hline K31 & - & - & - & - & 0.0842 \\
\hline K32 & - & - & - & - & 0.0699 \\
\hline
\end{tabular}

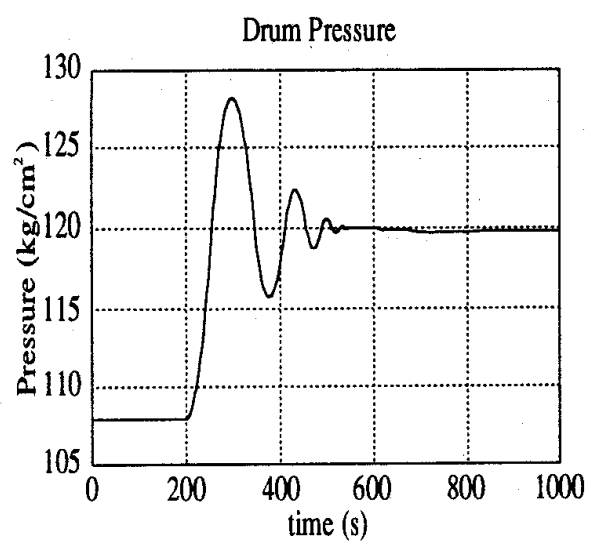

Fig. 2. GA/PI pressure response.

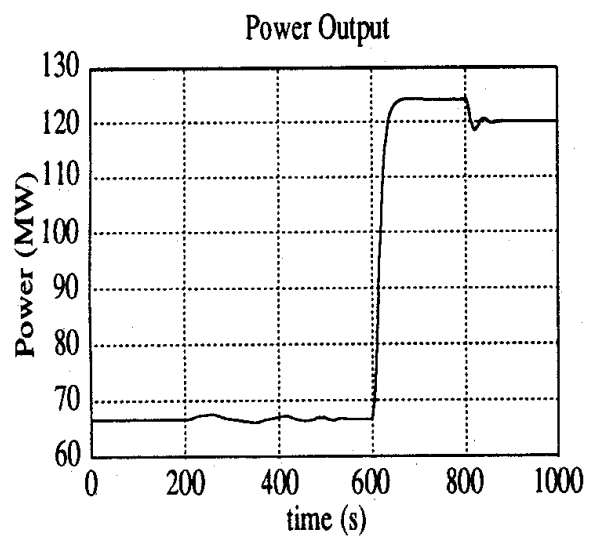

Fig. 3. GA/PI power response. change at 200 seconds when the level exceeds 0.15 meters from the nominal level. The steady-state error for the level is 0.000243 meters.

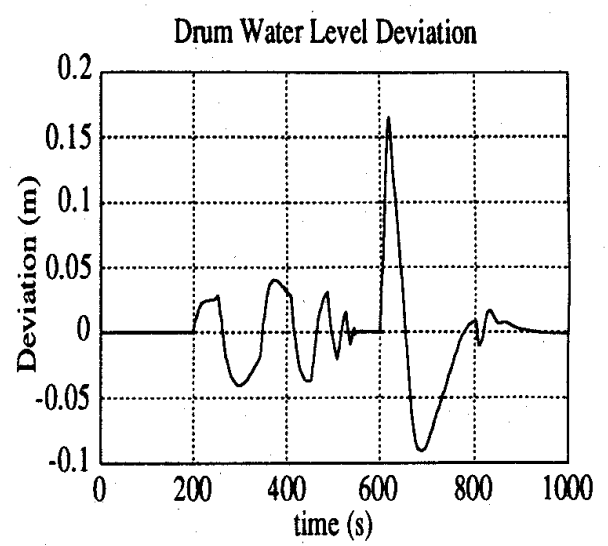

Fig. 4. GA/PI level response.

\section{B. GA/LQR Controller Results}

The performance of the system with the GA-designed LQR controller is shown in Figs. 5-7. The result of the GA/LQR design is the state feedback matrix,

$$
K=\left(\begin{array}{ccc}
0.0354 & 0.2236 & -0.1451 \\
-0.0430 & 0.0588 & 0.0039 \\
0.1609 & -0.6316 & 0.4275
\end{array}\right)
$$

It should be noted that, to ensure steady-state tracking, that a feedforward gain matrix is added to the system. This is obtained by calculating the system transfer function and finding a suitable matrix, F, so that the system tracks step demands in the steady-state. The values of the matrix are found to be [13]

$$
F=\left(\begin{array}{ccc}
0.0341 & 0.0018 & 0.6567 \\
-0.0051 & 0.0903 & -1.3537 \\
-0.4234 & -0.4088 & 94.3416
\end{array}\right)
$$

The pressure tracks the initial demand change well and there is a $5 \mathrm{~kg} / \mathrm{cm}^{2}$ pressure bump when the power demand is stepped up. The steady-state error in pressure is $0.252 \mathrm{~kg} / \mathrm{cm}^{2}$. The power response shows a large drop (25 MW) when the pressure demand is stepped up at 200 seconds. The power tracks the step demand at 600 seconds well achieving a steadystate error of $0.68 \mathrm{MW}$. The drum water level deviation undergoes relatively large deflections at each demand change in pressure and power. The largest of these is a level drop of 0.25 meters. The steady-state error in level is 0.0425 meters, due in large part from the deviation from the nominal operating point. 


\section{Optimal Controller Results}

Figs. 8-10 illustrate the performance of the standard LQR optimal controller. This controller is of state-feedback form that is designed from the model linearized at the $100 \%$ nominal operating point. The controller is developed using

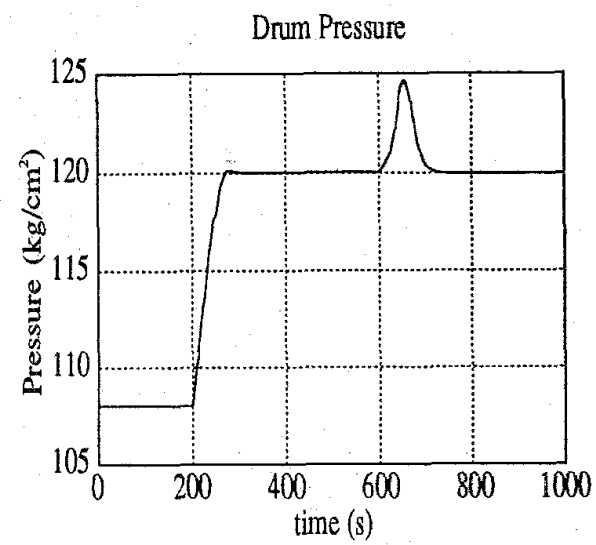

Fig. 5. GA/LQR pressure response.

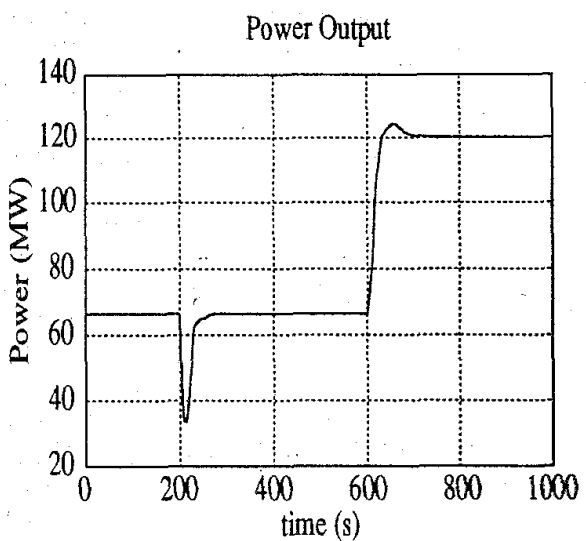

Fig. 6. GA/LQR power response.

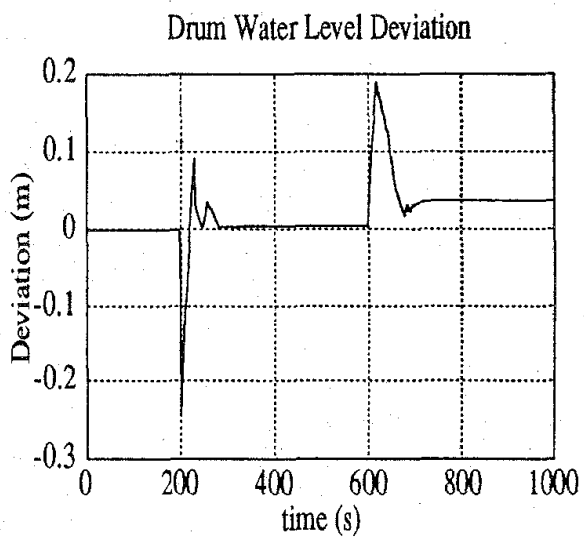

Fig. 7. GA/LQR level response. the following infinite time horizon quadratic performance index with the same state and input weighting matrices as discussed in section III.B:

$$
\mathrm{J}=\frac{1}{2} \int_{0}^{\infty}\left[\mathrm{x}^{\mathrm{T}} \mathrm{Qx}+\mathrm{u}^{\mathrm{T}} \mathrm{Ru}\right] \mathrm{dt}
$$

The result of the design is the state feedback matrix,

$$
K=\left(\begin{array}{ccc}
0.0359 & 0.0011 & 0.0031 \\
-0.0057 & 0.0770 & -0.0064 \\
-0.0413 & 0.0232 & 0.4444
\end{array}\right)
$$

The drum pressure shows good tracking at the first pressure demand change and a small bump of $1.5 \mathrm{~kg} / \mathrm{cm}^{2}$ at the power demand change at 600 seconds. The steady-state error in pressure is $0.046 \mathrm{~kg} / \mathrm{cm}^{2}$. The power output tracks the demand very well at both the pressure demand change and the power demand change. The steady-state error in power is $0.34 \mathrm{MW}$. The drum water level deviation shows a persistent chattering beginning at the pressure demand change at 200 seconds. This is a non-linear effect due to the saturation and rate limiting of the plant inputs. Examination of the poles of the system linearized at this particular operating point support that this is a non-linear effect since the poles lie in the left half of the complex plane. The persistent chattering in this output is detrimental to the actuators and is highly undesirable in a control system. We expect that the optimal control system would not be optimal at this deviation from the nominal operating point at which it was designed.

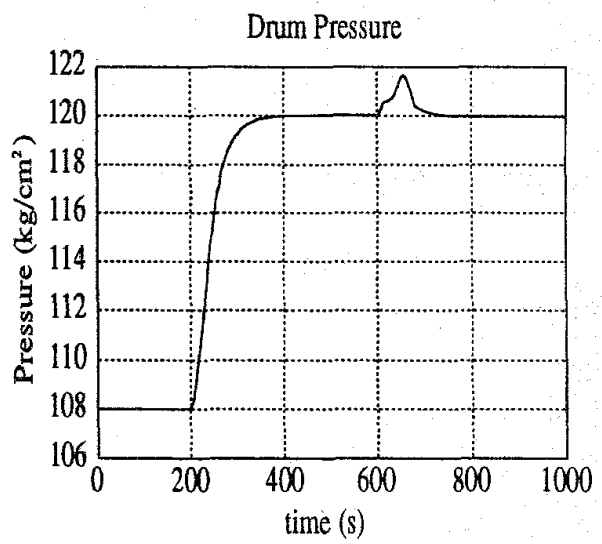

Fig. 8. LQR pressure response. 


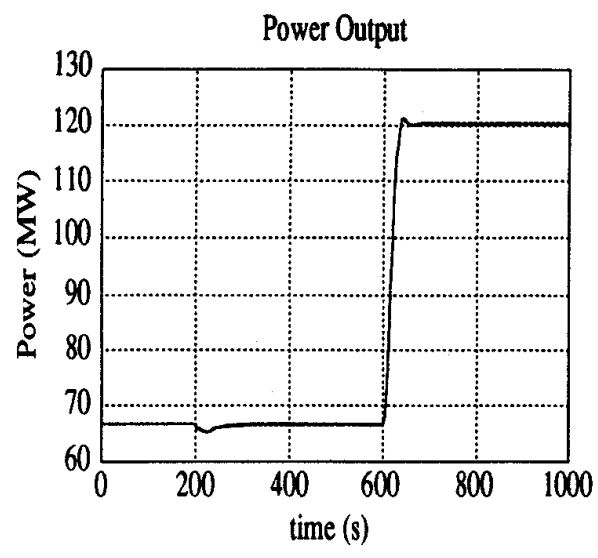

Fig. 9. LQR power response.

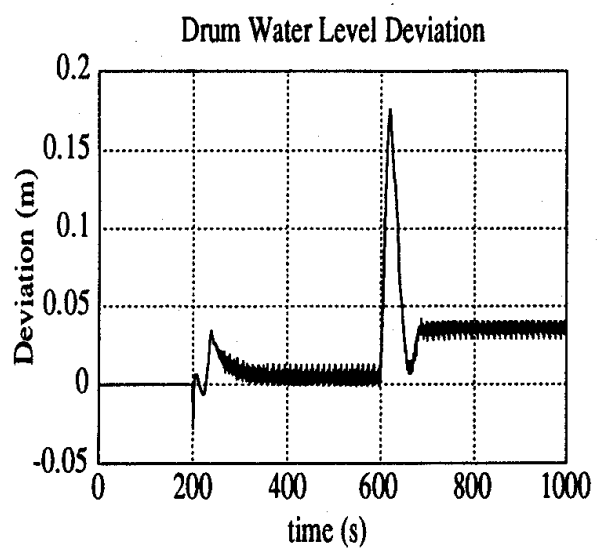

Fig. 10. LQR level response.

\section{Conclusions}

In this paper, a control system design methodology for a boiler-turbine plant was presented. The non-linear, MIMO plant was discussed as well as the associated linearized plant model. A brief summary of the GA was presented and its application to power plant control system design was discussed. The results of the GA design of a coupled PI controller and state feedback controller were presented. These results were compared to the results of a standard linear quadratic regulator control system. It was found that the GA/PI control system achieved good steady-state tracking but oscillations due to the integral action were prevalent. The GA/LQR control system performed well but at the cost of small but finite steady-state error.

\section{Acknowledgement}

The work was supported in part by a NSF grant (EID9212132), Research and Curriculum Development for Power Plant Intelligent Distributed Control, and NSF/EPRI joint project (ECS-9216504/RP-8030-4), Experimental Development of Power Reactor Intelligent Control.

\section{REFERENCES}

[1] A. Ben-Abdennour, K.Y. Lee, and R.M. Edwards, "Multivariable robust control of a power plant deaerator," IEEE Transactions on Energy Conversion, 8, pp. 123-129, March 1993.

[2] I.G. Klefenz, Automatic Control of Steam Power Plants, $3^{r d}$ Edition, Bibliographisches Institut, 1986.

[3] W.H. Kwon, S.W. Kim, P. Park, and E. Kim, "Design of a boiler-turbine control system using a modified LQG/LTR method," KIEE Transactions, 39, pp. 199. 209, February 1990.

[4] D.E. Goldberg, Genetic Algorithms in Search, Optimization, \& Machine Learning, Addison-Wesley, 1989.

[5] R.M. Brady, "Optimization strategies gleaned from biological evolution," Nature, 317, pp. 804-806, 1985.

[6] K. Krishnakumar and D.E. Goldberg, "Control system optimization using genetic algorithms," Journal of Guidance, Control, and Dynamics, 15, No. 3, pp. 735 $740,1992$.

[7] R.D. Bell and K.J. Aström, "Dynamic models for boilerturbine-alternator units: Data logs and parameter estimation for a 160 MW unit," Report TFRT-3192, Lund Institute of Technology, Sweden, 1987.

[8] K.J. Åström and K. Eklund, "A simplified non-linear model of a drum-boiler-turbine unit," International Journal of Control, 16, pp. 146-169, 1972.

[9] A.J. Morton and P.H. Price, "The controllability of steam output, pressure and water level in drum boilers," Steam at Work: Industrial and Marine Steam Plant, pp. 75-84, 1977.

[10] John H. Holland, Adaptation in Natural and Artificial Systems, MIT Press, 1992.

[11] J. Koza, Genetic Programming: On the Programming of Computers by Means of Natural Selection, MIT Press, 1992.

[12] L. Davis (Ed.), Genetic Algorithms and Simulated Annealing, Morgan Kaufmann, 1987.

[13] H. Kwakernaak and R. Sivan, Linear Optimal Control Systems, John Wiley and Sons, 1972. 